\title{
An alternative approach to extending pseudo-Anosovs over compression bodies
}

\author{
ROBERT ACKERMANN
}

\begin{abstract}
Biringer, Johnson, and Minsky proved that any pseudo-Anosov whose stable lamination is the limit of disks in a compression body has a power which extends over some non-trivial minimal compression body. This paper presents an alternative proof of their theorem. The key ingredient is the existence of a certain collection of disks whose boundaries are formed from an arc of the stable lamination and an arc of the unstable lamination. The proof here also shows that there are only finitely many minimal compression bodies over which a power of a pseudo-Anosov can extend.
\end{abstract}

57M99

\section{Introduction}

The following theorem was proved by Biringer, Johnson, and Minsky.

Theorem 1.1 [1, Theorem 1.1] Let $\varphi: F \rightarrow F$ be a pseudo-Anosov with stable lamination $\mathcal{L}^{+}$and unstable lamination $\mathcal{L}^{-}$. Suppose also that a lamination $K^{+} \supseteq \mathcal{L}^{+}$ bounds in a compression body $M$, and $M$ is minimal with respect to this condition. Then there exists $k$ such that $\varphi^{k}$ extends over $M$.

Here we say that a lamination bounds if it is the Hausdorff limit of curves bounding disks in the compression body. A compression body $M$ is minimal with respect to the condition that $K^{+}$bounds if there is no inequivalent $N \subset M$ in which $K^{+}$bounds. Their proof makes use of relatively recent ideas including $\delta$-hyperbolic geometry, the curve complex, and Ahlfors-Bers theory. They also give examples which show that their theorem is false if $\varphi^{k}$ is replaced with $\varphi$ in the conclusion.

The purpose of this paper is to offer an alternative proof to this theorem using older ideas first introduced by Casson and Long. More specifically, Casson and Long in [4] provide an algorithm for determining whether a particular pseudo-Anosov extends over some compression body, and Long in [6] goes on to show that a pair of minimal, transverse laminations can bound in only finitely many compression bodies. 
The proof given here is achieved by generalizing lemmas of Casson and Long. The basic idea is to show that disks of a particular type must exist in any compression body in which the stable lamination of $\varphi$ bounds and some curve approximating the unstable lamination bounds as well. Using these disks, we build a non-empty but finite collection of compression bodies over which $\varphi$ could potentially extend. Within this collection there is a (possibly smaller) collection which is invariant under the action of $\varphi$, implying that a power of $\varphi$ extends.

\section{Definitions and basic facts}

Let $F$ be a closed, orientable surface of genus at least two. A compression body is any 3-manifold formed by taking $F \times I$, attaching disjoint 2-handles to the boundary surface $F \times\{1\}$, and filling in any resulting 2 -spheres with 3 -handles. The boundary surface $F \times\{0\}$ is called the exterior surface of $M$ which we will denote $\partial_{E} M$. Call $F \times I$ the trivial compression body.

A compression body $M$ with exterior surface $\partial_{E} M$ has associated to it a normal subgroup $N=\operatorname{ker}\left(i_{*}: \pi_{1}\left(\partial_{E} M\right) \rightarrow \pi_{1}(M)\right)$, where $i: \partial_{E} M \rightarrow M$ is inclusion. $N$ is equal to the image of the fundamental group of a regular covering of $\partial_{E} M$ which is planar, so we call $N$ the planar kernel of $M$ (see [6]).

Given a fixed surface $F$ and collections of disjoint, non-isotopic, essential curves $\mathcal{C}_{1}, \mathcal{C}_{2}$, we say that the pairs $\left(F, \mathcal{C}_{1}\right)$ and $\left(F, \mathcal{C}_{2}\right)$ are equivalent if $\left\langle\mathcal{C}_{1}\right\rangle_{N}=\left\langle\mathcal{C}_{2}\right\rangle_{N}$, where $\left\langle\mathcal{C}_{i}\right\rangle_{N}$ denotes the normal closure of the subgroup generated by $\mathcal{C}_{i}$ in $\pi_{1}(F)$. If $M$ is a compression body and $\theta: F \rightarrow \partial_{E} M$ is a homeomorphism such that $\theta_{*}\langle\mathcal{C}\rangle_{N}=\operatorname{ker}\left(i_{*}: \pi_{1}\left(\partial_{E} M\right) \rightarrow \pi_{1}(M)\right)$ we say that $M$ is built from $(F, \mathcal{C})$.

A geodesic lamination $\mathcal{L}$ on $F$ is a closed subset which can be written as the union of disjoint geodesic leaves. A geodesic lamination is minimal if the closure of any leaf is the whole lamination, and a geodesic lamination fills if each component of $F \backslash \mathcal{L}$ is simply connected. We call the closure of these components the complementary regions of $\mathcal{L}$, and say $\mathcal{L}$ is maximal if every complementary region is an ideal triangle. To save words, a lamination will always be assumed to be a geodesic lamination.

Given a closed surface $F$, the Hausdorff metric is a metric on all closed subsets of $F$. For two closed subsets $A$ and $B$, distance is defined by $d_{H}(A, B) \leq \epsilon$ if there are $\epsilon$-neighborhoods $N_{\epsilon}(A) \subseteq B$ and $N_{\epsilon}(B) \subseteq A$. The Hausdorff metric is particularly useful for measuring how close a simple closed curve is to a minimal lamination.

A surface automorphism $\varphi: F \rightarrow F$ is called pseudo-Anosov if it preserves a pair of transverse measured laminations $\left(\mathcal{L}^{+}, \mu_{+}\right)$and $\left(\mathcal{L}^{-}, \mu_{-}\right)$, called the stable and 
unstable lamination respectively. In this case, both $\mathcal{L}^{+}$and $\mathcal{L}^{-}$are minimal and filling. We say that $\varphi$ extends over a compression body $M$ built from $(F, \mathcal{C})$ if there is an automorphism $\psi: M \rightarrow M$ such that $\left.\psi\right|_{\partial_{E} M}=\theta \varphi \theta^{-1}$. A necessary and sufficient condition for an automorphism to extend is that $(F, \mathcal{C})$ and $(F, \varphi \mathcal{C})$ are equivalent (see [3, Lemma 5.2]).

An important fact about the behavior of pseudo-Anosovs is that they exhibit source-sink dynamics on the space of all measured laminations. In particular, suppose that $\varphi$ is a pseudo-Anosov with invariant laminations $\mathcal{L}^{+}$and $\mathcal{L}^{-}$. If $\mathcal{L}^{+}$and $\mathcal{L}^{-}$are maximal and $\mathcal{L}$ is a third lamination not equal to $\mathcal{L}^{+}$or $\mathcal{L}^{-}$, then the sequence $\left\{\varphi^{k}(\mathcal{L})\right\}$ converges to $\mathcal{L}^{+}$and $\left\{\varphi^{-k}(\mathcal{L})\right\}$ converges to $\mathcal{L}^{-}$in the Hausdorff topology (see [2]).

Crucial to our discussion is the following definition, inspired from [4].

Definition 2.1 Let $\mathcal{L}$ be a geodesic lamination in a surface $F$. Then $\mathcal{L}$ bounds in the pair $(F, \mathcal{C})$ if there is a sequence of simple closed curves $\left\{C_{i}\right\}$ such that:

(1) The element of $\pi_{1} F$ corresponding to $C_{i}$ is in $\langle\mathcal{C}\rangle_{N}$ for all $i$.

(2) $C_{i} \rightarrow \mathcal{L}$ as $i \rightarrow \infty$ in the Hausdorff metric.

If $M$ is a compression body built from $(F, \mathcal{C})$ and $\mathcal{L}$ bounds, then the corresponding lamination isotopic to $\theta(\mathcal{L})$ in $\partial_{E} M$ has a sequence of simple closed curves $\left\{D_{i}\right\}$ all of which bound disks in $M$ such that $D_{i} \rightarrow \mathcal{L}$ as $i \rightarrow \infty$. In this case we will also say $\mathcal{L}$ bounds in $M$. Note that if $\mathcal{L}^{+}$and $\mathcal{L}^{-}$are transverse, minimal, and maximal measured laminations with full support then condition (2) is equivalent to $\mu_{+}\left(C_{i}\right) \rightarrow 0$ as $i \rightarrow \infty$ (possibly after isotopy). In [1], a similar notion of bounding is expressed in terms of limit sets. One motivation for this definition is that if a pseudo-Anosov extends then both its stable and unstable lamination must bound, by source-sink dynamics.

If $\mathcal{L}$ is a lamination, then we say that $(F, \mathcal{C})$ is minimal with respect to $\mathcal{L}$ bounding if $\mathcal{L}$ bounds in $(F, \mathcal{C})$ and if $(F, \mathcal{D})$ is another pair with $\langle\mathcal{D}\rangle_{N} \subset\langle\mathcal{C}\rangle_{N}$ then $\mathcal{L}$ does not bound in $(F, \mathcal{D})$. Similarly, we say that $(F, \mathcal{C})$ is minimal with respect to a collection of laminations $\mathcal{A}$ bounding if $\langle\mathcal{C}\rangle_{N}$ is the "smallest" normal subgroup in which every element of $\mathcal{A}$ bounds.

\section{Disks in compression bodies}

Throughout take $\left(\mathcal{L}^{+}, \mu_{+}\right)$and $\left(\mathcal{L}^{-}, \mu_{-}\right)$to be transverse, minimal, and maximal measured laminations in the exterior surface of some compression body $M$. In this section, we construct a collection of bounding curves built from "short" arcs of $\mathcal{L}^{-}$ 
and "long" arcs of $\mathcal{L}^{+}$with controlled $\mu_{-}-$measure. Necessary for the existence of these curves is that $\mathcal{L}^{+}$bounds and some curve $C$ approximating $\mathcal{L}^{-}$bounds as well. We begin by stating a lemma first proved in [6].

Lemma 3.1 Let $\epsilon>0$ be given. Then there are numbers $M(\epsilon), m(\epsilon)$ such that:

(1) If $\alpha^{+} \subseteq \mathcal{L}^{+}, \alpha^{-} \subseteq \mathcal{L}^{-}$are arcs with $\mu_{-}\left(\alpha^{+}\right)>M$ and $\mu_{+}\left(\alpha^{-}\right)>\epsilon$, then int $\alpha^{+} \cap$ int $\alpha^{-} \neq \varnothing$.

(2) If $\alpha^{+} \subseteq \mathcal{L}^{+}, \alpha^{-} \subseteq \mathcal{L}^{-}$are arcs with $\mu_{+}\left(\alpha^{-}\right)<\epsilon$ and $\mid$ int $\alpha^{+} \cap$ int $\alpha^{-} \mid \geq 2$, then $\mu_{-}\left(\alpha^{+}\right)>m$.

Furthermore, $M(\epsilon), m(\epsilon) \rightarrow \infty$ as $\epsilon \rightarrow 0$.

Let $N_{\delta}\left(\mathcal{L}^{-}\right)$be a closed $\delta$-neighborhood of $\mathcal{L}^{-}$. Such a neighborhood can be foliated by intervals so that it has the structure of a product, and each tie $t$ can be thought of as an arc in $F$ transverse to $\mathcal{L}^{-}$. Let $r=r(\delta)=\max \left\{\mu_{-}(t) \mid t\right.$ is a tie of $\left.N_{\delta}\left(\mathcal{L}^{-}\right)\right\}$.

Lemma 3.2 Let $C$ be a geodesic simple closed curve such that $d_{H}\left(C, \mathcal{L}^{-}\right)<\delta$ for some small $\delta>0$, and suppose that $\left\{A_{n}\right\}$ is a sequence of simple closed geodesics converging to $\mathcal{L}^{+}$. Then for any $\epsilon>0$, there is an $N$ such that for all $n \geq N$ we have:

(1) If $\alpha^{+} \subseteq A_{n}, \alpha^{-} \subseteq C$ are arcs with $\mu_{-}\left(\alpha^{+}\right)>2 M$ and $\mu_{+}\left(\alpha^{-}\right)>\epsilon$, then int $\alpha^{+} \cap$ int $\alpha^{-} \neq \varnothing$.

(2) If $\alpha^{+} \subseteq A_{n}, \alpha^{-} \subseteq C$ are arcs with $\mu_{+}\left(\alpha^{-}\right)<\epsilon$ and $\mid$ int $\alpha^{+} \cap$ int $\alpha^{-} \mid \geq 2$, then $\mu_{-}\left(\alpha^{+}\right)>m-2 r$, where $r=r(\delta)$ as above.

Proof Given an arc $\alpha^{-} \subseteq C$, shrink it slightly and assume its endpoints are on leaves of $\mathcal{L}^{+}$(without changing $\mu_{+}\left(\alpha^{-}\right)$). Then since $\delta>0$ is small, we can slide its endpoints along leaves of $\mathcal{L}^{+}$to obtain a nearby arc $\beta \subseteq \mathcal{L}^{-}$with $\mu_{+}(\beta)=\mu_{+}\left(\alpha^{-}\right)$.

Choose $N$ such that for all $n \geq N$ the curve $A_{n}$ satisfies:

(1) Lemma 3.1 holds with $\operatorname{arcs}$ of $A_{n}$ in place of $\operatorname{arcs}$ in $\mathcal{L}^{+}$.

(2) If $\alpha^{-} \subseteq C$ is an arc and $\beta \subseteq \mathcal{L}^{-}$is chosen as above, then for any $p \in \operatorname{int} \beta \cap A_{n}$ there is an $\operatorname{arc} \phi \subseteq A_{n}$ with endpoints on $\beta$ and $\alpha^{-}$(one of these is $p$ ) with $\mu_{-}(\phi)<r=r(\delta)$.

These conditions can always be satisfied, after possibly a small isotopy of $\beta$, because the angles between nearby geodesics are close in a lamination (see [2]), and because the measure of an arc is preserved under homotopy respecting the leaves of $\mathcal{L}^{-}$. 
To prove the first conclusion, let $n \geq N$ and take any $\operatorname{arc} \alpha^{+} \subseteq A_{n}$ with $\mu_{-}\left(\alpha^{+}\right)>2 M$. For the sake of contradiction suppose that there is an arc $\alpha^{-} \subseteq C$ with $\mu_{+}\left(\alpha^{-}\right)>\epsilon$ such that int $\alpha^{+} \cap$ int $\alpha^{-}=\varnothing$. Take $\beta \subseteq \mathcal{L}^{-}$as above and note that by condition (1), $\alpha^{+}$must intersect $\beta$ at least twice. Thus by condition (2) both endpoints of $\alpha^{+}$must lie on short arcs of $A_{n}$ with endpoints on int $\beta$ and int $\alpha^{-}$. But then by shrinking $\alpha^{+}$ and allowing $\mu_{-}\left(\alpha^{+}\right)$to change by at most $2 r<M$ we obtain an arc which does not intersect $\beta$, a contradiction. Thus int $\alpha^{+} \cap$ int $\alpha^{-} \neq \varnothing$.

For the second conclusion, again fix $n \geq N$ and suppose $\alpha^{-} \subseteq C, \alpha^{+} \subseteq A_{n}$ are arcs with $\mu_{+}\left(\alpha^{-}\right)<\epsilon$ and $\mid$ int $\alpha^{+} \cap$ int $\alpha^{-} \mid \geq 2$. Again take $\beta \subseteq \mathcal{L}^{-}$as above. Then by condition (2), $\alpha^{+}$can be extended to an arc that intersects int $\beta$ at least twice with $\mu_{--}$ measure at most $2 r$ more than $\mu_{-}\left(\alpha^{+}\right)$. Thus by Lemma 3.1, $\mu_{-}\left(\alpha^{+}\right)>m-2 r$.

The next lemma mirrors the proof of [6, Lemma 2.4] and relies on Lemma 3.2 to control the lengths of arcs.

Lemma 3.3 Let $\delta>0$ be small and let $C$ be a geodesic simple closed curve with $d_{H}\left(C, \mathcal{L}^{-}\right)<\delta$. Suppose in addition that $C$ and $\mathcal{L}^{+}$both bound in a compression body $M$. Then for any small $\epsilon>0$ there are arcs $\alpha^{+} \subseteq \mathcal{L}^{+}, \alpha^{-} \subseteq \mathcal{L}^{-}$such that $\alpha^{+} \cup \alpha^{-}$is the boundary of a disk, and:

(1) $\mu_{+}\left(\alpha^{-}\right) \leq \epsilon$.

(2) $m(2 \epsilon)-2 r \leq \mu_{-}\left(\alpha^{+}\right) \leq 2 M(\epsilon)+2 r$, where $r=r(\delta)$.

Proof Since $\mathcal{L}^{+}$bounds, there is a sequence of closed geodesics $\left\{A_{n}\right\}$ all bounding disks in $M$ and converging to $\mathcal{L}^{+}$. Choose $N$ as in Lemma 3.2 and let $A=A_{n}$ for some $n \geq N$. Let $D^{-}$be the disk with boundary $C$, and $D^{+}$the disk with boundary $A$. We assume $\epsilon$ is very small compared to the $\mu_{+}$-measure of $C$.

After isotopy, $D^{+} \cap D^{-}$is a collection of arcs with endpoints on $A \cap C$. We say that an $\operatorname{arc} \gamma$ on $C$ or $A$ contains a complete set if whenever one endpoint of an arc in $D^{+} \cap D^{-}$is on $\gamma$, the other endpoint is on $\gamma$ as well. Choose an arc $\gamma^{+} \subseteq A$ such that $\gamma^{+}$is complete, $\mu_{-}\left(\gamma^{+}\right) \geq 2 M$, and $\gamma^{+}$is minimal with respect to these conditions. By Lemma 3.2, $\gamma^{+}$exists and intersects $C$ many times.

Choose an $\operatorname{arc} \phi \subseteq D^{+} \cap D^{-}$such that $\phi$ has endpoints on int $\gamma^{+}$and $\phi$ is an outermost such arc in $D^{-}$. Then choose $\gamma^{-} \subseteq C=\partial D^{-}$to be the arc with endpoints equal to the endpoints of $\phi$ and with the property that int $\gamma^{-} \cap$ int $\gamma^{+}=\varnothing$. By Lemma 3.2, $\mu_{+}\left(\gamma^{-}\right)<\epsilon$.

Now set $\beta^{+} \subseteq A$ to be the sub-arc of $\gamma^{+}$having endpoints $\partial \gamma^{-}$(which equals $\partial \phi$ ), and note that int $\beta^{+} \cap$ int $\gamma^{-}=\varnothing$. We can stretch $\beta^{+}$and $\gamma^{-}$a small amount so that $\mid$ int $\beta^{+} \cap$ int $\gamma^{-} \mid \geq 2$ and hence $\mu_{-}\left(\beta^{+}\right) \geq m(2 \epsilon)-2 r$. 
Since $\partial \beta^{+}=\partial \phi \subseteq$ int $\gamma^{+}$, the arc $\beta^{+}$is a complete, proper sub-arc of $\gamma^{+}$. Thus $\mu_{-}\left(\beta^{+}\right) \leq 2 M$ by minimality of $\gamma^{+}$.

Now slide $\gamma^{-}$along the leaves of $\mathcal{L}^{+}$to an arc $\alpha^{-}$with $\mu_{+}\left(\alpha^{-}\right)=\mu_{+}\left(\gamma^{-}\right)<\epsilon$ and endpoints on leaves of $\mathcal{L}^{+}$. Isotopic to $\gamma^{+}$is an $\operatorname{arc} \alpha^{+} \subseteq \mathcal{L}^{+}$with $\partial \alpha^{+}=\partial \alpha^{-}$and $\left|\mu_{-}\left(\gamma^{+}\right)-\mu_{-}\left(\alpha^{+}\right)\right| \leq r$. The curve $\alpha^{+} \cup \alpha^{-}$is essential because it is the union of geodesic arcs, and is isotopic to the boundary of the disk formed by gluing pieces of $D^{+}$and $D^{-}$cut out by $\phi$.

\section{Finitely many minimal compression bodies}

Recall that $(F, \mathcal{C})$ is minimal with respect to a lamination $\mathcal{L}$ bounding if $\mathcal{L}$ bounds in $(F, \mathcal{C})$ and whenever $(F, \mathcal{D})$ is another pair with $\langle\mathcal{D}\rangle_{N} \subset\langle\mathcal{C}\rangle_{N}$ then $\mathcal{L}$ does not bound in $(F, \mathcal{D})$. Also note that a single geodesic simple closed curve is a lamination.

Lemma 4.1 [5] Let $\mathcal{K}$ be any finite collection of simple closed curves. Then there are at most finitely many pairs $(F, \mathcal{C})$ which are minimal with respect to $\mathcal{K}$.

The next lemma gives even more control over what compression bodies can contain a specified collection of disks (this is referred to as a "folklore lemma" in [6]).

Lemma 4.2 Let $\left\{\left(F, \mathcal{C}_{1}\right), \ldots,\left(F, \mathcal{C}_{k}\right)\right\}$ be pairwise inequivalent, and suppose also that $\left\langle\mathcal{C}_{1}\right\rangle_{N} \subset\left\langle\mathcal{C}_{2}\right\rangle_{N} \subset \cdots \subset\left\langle\mathcal{C}_{k}\right\rangle_{N}$ where inclusions are strict. Then there is an integer $P$, depending only on the genus of $F$, such that $k \leq P$.

The following is a generalization of lemmas first proved in [2] and [6], and is one of the key ingredients in proving our main result.

Lemma 4.3 Let $\left\{\mathcal{K}_{i}\right\}$ be a sequence of finite collections of essential simple closed curves such that any sequence $\left\{C_{i} \mid C_{i} \in \mathcal{K}_{i}\right\}$ converges to a lamination $\mathcal{L}$. Let $\mathcal{M}$ be the collection of all pairwise inequivalent pairs $(F, \mathcal{C})$ minimal with respect to $\mathcal{L}$ bounding and in which a sequence $\left\{C_{i} \mid C_{i} \in \mathcal{K}_{i}\right\}$ bounds. Then $\mathcal{M}$ is finite.

Proof Let $\mathcal{P}$ be the collection of all pairwise inequivalent pairs $(F, \mathcal{C})$ which are minimal with respect to some finite (or empty) collection $\left\{C_{1}, \ldots, C_{n} \mid C_{i} \in \mathcal{K}_{i}\right\}$, and where either $\mathcal{L}$ does not bound in $(F, \mathcal{C})$ or if it does then $(F, \mathcal{C})$ is minimal. We consider $\mathcal{P}$ as a partially ordered set with $(F, \mathcal{D}) \leq(F, \mathcal{C})$ if $\langle\mathcal{D}\rangle_{N} \subseteq\langle\mathcal{C}\rangle_{N}$. Note that the trivial pair $(F, \varnothing)$ is the unique least element of $P$. To save on notation, let $\Delta_{n}$ formally denote a collection $\left\{C_{1}, \ldots, C_{n} \mid C_{i} \in \mathcal{K}_{i}\right\}$. 
Any $(F, \mathcal{C}) \in \mathcal{M}$ must be minimal with respect to some $\left\{C_{i} \mid C_{i} \in \mathcal{K}_{i}\right\}$, since any pair in which such a sequence bounds has $\mathcal{L}$ bounding as well. Thus, by Lemma 4.2, any $(F, \mathcal{C}) \in \mathcal{M}$ must be minimal with respect to some finite collection $\Delta_{n}$ and hence $\mathcal{M} \subseteq \mathcal{P}$.

We show that $\mathcal{P}$ is finite. By Lemma 4.2, any chain $\left(F, \mathcal{C}_{1}\right) \subset\left(F, \mathcal{C}_{2}\right) \subset \cdots$ in $\mathcal{P}$ is finite, so it only remains to show that for every $(F, \mathcal{C})$ there are finitely many $(F, \mathcal{D})$ such that whenever $(F, \mathcal{C}) \subseteq X \subseteq(F, \mathcal{D})$ we have $X=(F, \mathcal{C})$ or $X=(F, \mathcal{D})$. Call such a pair $(F, \mathcal{D})$ a direct descendant of $(F, \mathcal{C})$.

Suppose that $(F, \mathcal{C}) \in \mathcal{P}$ is minimal for some $\Delta_{n}$ and that $\mathcal{L}$ does not bound in $(F, \mathcal{C})$. Then there is a minimal $R \in \mathbb{N}$ such that no collection $\Delta_{R} \supset \Delta_{n}$ bounds in $(F, \mathcal{C})$. By Lemma 4.1, there are only finitely many compression bodies minimal with respect to a collection $\Delta_{l}$ with $l \leq R$. Any direct descendant of $(F, \mathcal{C})$ must be minimal with respect to one of these collections, and thus $(F, \mathcal{C})$ has only finitely many direct descendants.

Now suppose $(F, \mathcal{C}) \in \mathcal{P}$ is such that an infinite sequence $\left\{C_{i} \mid C_{i} \in \mathcal{K}_{i}\right\}$ bounds. Then $\mathcal{L}$ bounds in $(F, \mathcal{C})$, and by minimality $(F, \mathcal{C})$ has no direct descendants in this case. Thus $\mathcal{P}$ is finite, and $\mathcal{M}$ is finite as well.

Recall that $\varphi$ extends over a compression body built from $(F, \mathcal{C})$ if and only if $(F, \mathcal{C})$ and $(F, \varphi \mathcal{C})$ are equivalent.

Lemma 4.4 Let $\varphi$ be a pseudo-Anosov with maximal stable, unstable laminations $\mathcal{L}^{+}, \mathcal{L}^{-}$and say that $\mathcal{L}^{+}$bounds in some $(F, \mathcal{C})$. Let $\delta>0$ and let $\mathcal{N}$ be the collection of all pairs $(F, \mathcal{D})$ minimal with respect to $\mathcal{L}^{+}$and which have a curve $D$ in $\langle\mathcal{D}\rangle_{N}$ with $d_{H}\left(D, \mathcal{L}^{-}\right)<\delta$. Then $\mathcal{N}$ is non-empty.

Proof Let $(F, \mathcal{C})$ be any pair minimal for $\mathcal{L}^{+}$and suppose that $C$ is a curve in $\langle\mathcal{C}\rangle_{N}$. By the source-sink dynamics of pseudo-Anosovs, for some $k$ the curve $\varphi^{-k}(C)$ is, after isotopy, a geodesic simple closed curve with $d_{H}\left(\varphi^{-k}(C), \mathcal{L}^{-}\right)<\delta$.

Now, let $\left\{C_{i}\right\}$ be a sequence of curves in $\langle\mathcal{C}\rangle_{N}$, such that $\left\{C_{i}\right\}$ approaches $\mathcal{L}^{+}$in the Hausdorff topology. Then $\left\{\varphi^{-k}\left(C_{i}\right)\right\}$ has the same property in $\left(F, \varphi^{-k} \mathcal{C}\right)$. Furthermore, $\left(F, \varphi^{-k} \mathcal{C}\right)$ is still minimal, for if $\langle\mathcal{D}\rangle_{N} \subseteq\left\langle\varphi^{-k} \mathcal{C}\right\rangle_{N}$ is such that $\mathcal{L}^{+}$bounds in $(F, \mathcal{D})$, then $\left\langle\varphi^{k} \mathcal{D}\right\rangle_{N} \subseteq\langle\mathcal{C}\rangle_{N}$ and in fact $\left(F, \varphi^{k} \mathcal{D}\right)$ is equivalent to $(F, \mathcal{C})$ by minimality.

Finally, we prove the main theorem. 
Theorem 4.5 Let $\varphi: F \rightarrow F$ be a pseudo-Anosov with stable lamination $\mathcal{L}^{+}$. Assume that $\mathcal{L}^{+}$is maximal, that $\mathcal{L}^{+}$bounds in $(F, \mathcal{C})$, and that $(F, \mathcal{C})$ is minimal with respect to this condition. Then there exists $k$ such that $\varphi^{k}$ extends over a compression body $M$ built from $(F, \mathcal{C})$.

Proof Choose $M$ and $\theta: F \rightarrow M$ such that $\theta$ takes geodesics to geodesics and preserves the transverse measures of curves. Let $\delta>0$ be small and choose a decreasing sequence $\left\{\epsilon_{i}\right\}$ with $\epsilon_{1}>0$ and $\epsilon_{i} \rightarrow 0$ as $i \rightarrow \infty$. Define $\mathcal{K}_{i}$ to be the collection of all simple closed curves $\alpha^{+} \cup \alpha^{-}$formed from arcs $\alpha^{+} \subseteq \theta\left(\mathcal{L}^{+}\right)$and $\alpha^{-} \subseteq \theta\left(\mathcal{L}^{-}\right)$, where $m\left(2 \epsilon_{i}\right)-2 r \leq \mu_{-}\left(\alpha^{+}\right) \leq 2 M\left(\epsilon_{i}\right)+2 r$ and $\mu_{+}\left(\alpha^{-}\right) \leq \epsilon_{i}$ (here $M, m$, and $r=r(\delta)$ are as in Lemma 3.2). After identifying isotopic curves, each $\mathcal{K}_{i}$ is finite and any sequence $\left\{C_{i} \mid C_{i} \in \mathcal{K}_{i}\right\}$ converges to $\theta\left(\mathcal{L}^{+}\right)$. Furthermore, $\theta^{-1}\left(\mathcal{K}_{i}\right)$ gives collections of curves in $F$ with the same properties converging to $\mathcal{L}^{+}$.

Now let $\mathcal{N}$ be the collection of all pairs $(F, \mathcal{D})$ which are minimal for $\mathcal{L}^{+}$and also have a sequence of curves $\left\{C_{i} \mid C_{i} \in \theta^{-1}\left(\mathcal{K}_{i}\right)\right\}$ all of which are in $\langle\mathcal{D}\rangle_{N}$. By Lemma 4.3, $\mathcal{N}$ is finite.

Let $\mathcal{N}^{\prime}$ be the collection of pairs $(F, \mathcal{D})$ which are minimal for $\mathcal{L}^{+}$and also have a curve $C$ in $\langle\mathcal{D}\rangle_{N}$ with $d_{H}\left(C, \mathcal{L}^{-}\right)<\delta$. By Lemma 4.4, the set $\mathcal{N}^{\prime}$ is nonempty and by Lemma 3.3 it is contained in $\mathcal{N}$. Applying the techniques of Lemma 4.4 once again shows that $\varphi^{-t} \mathcal{N}^{\prime} \subseteq \mathcal{N}^{\prime}$ for some $t$, and thus there is a subset of $\mathcal{N}$ invariant under the action of $\varphi^{-1}$. Call this collection $\mathcal{N}^{*}$.

Now, $\left(F, \varphi^{-s} \mathcal{C}\right)$ lies in $\mathcal{N}^{*}$ and thus for some $k$ we have $\varphi^{-s-k}\langle\mathcal{C}\rangle_{N}=\varphi^{-s}\langle\mathcal{C}\rangle_{N}$. Composing with $\varphi^{s+k}$ we have $\langle\mathcal{C}\rangle_{N}=\varphi^{k}\langle\mathcal{C}\rangle_{N}$ and so $\varphi^{k}$ extends over $M$.

The proof implies the following corollary, though it also follows from results of [4].

Corollary 4.6 Let $\varphi$ be a pseudo-Anosov with maximal invariant laminations. Then $\varphi$ extends for at most finitely many inequivalent pairs $(F, \mathcal{C})$ which are minimal with respect to the condition that the stable lamination of $\varphi$ bounds.

Remark The observant reader will note that the theorem above is not quite the same as the theorem of Biringer, Johnson, and Minsky as we have added the hypothesis that the invariant laminations of $\varphi$ are maximal. If they are not, it is necessary to consider a finite collection of laminations which are formed from $\mathcal{L}^{+}$and $\mathcal{L}^{-}$by adding isolated leaves which "cut across" the diagonals of principal regions (see [2]). Lemmas 3.2, 3.3, and 4.4 can be modified to take into account this situation, however it makes their statements and proofs far more clumsy so we do not do so here. 


\section{References}

[1] I Biringer, J Johnson, Y Minsky, Extending pseudo-Anosov maps into compression bodies, J. Topol. 6 (2013) 1019-1042 MR3145149

[2] A J Casson, S A Bleiler, Automorphisms of surfaces after Nielsen and Thurston, London Math. Soc. Student Texts 9, Cambridge Univ. Press (1988) MR964685

[3] A J Casson, C M Gordon, A loop theorem for duality spaces and fibred ribbon knots, Invent. Math. 74 (1983) 119-137 MR722728

[4] A J Casson, D D Long, Algorithmic compression of surface automorphisms, Invent. Math. 81 (1985) 295-303 MR799268

[5] D D Long, Planar kernels in surface groups, Quart. J. Math. Oxford Ser. 35 (1984) 305-310 MR755667

[6] D D Long, Bounding laminations, Duke Math. J. 56 (1988) 1-16 MR932854

Department of Mathematics, University of California, Santa Barbara Santa Barbara, CA 93106-3080, USA

rackermann@math.ucsb.edu

Received: 24 June 2014 Revised: 6 November 2014 
